\title{
Autoinmunidad en pacientes con pérdida recurrente del embarazo
}

\author{
Francisco J. Espinel*; MD, Julio C. Mendoza**; MD, Bac. Jeannette Cubillos***; Bac. Emilce Botero****
}

\begin{abstract}
RESUMEN: Un grupo de 90 mujeres con antecedente de 2 o más abortos espontáneos de causa desconocida, sin historia de partos anteriores, con estudio inmunológico que determinó ausencia de respuesta aloinmune y un grupo de control de 31 mujeres con antecedente de fertilidad comprobada, se estudiaron para anticuerpos a antígenos nucleares, anticoagulante lúpico y fosfolípidos. Demostramos serológicamente un incremento en la respuesta autoinmune a partir del segundo aborto. Los anticuerpos antifosfolípidos y los anticuerpos antinucleares aparecen presentes ya como epifenómenos ya como factores etiológicos en pacientes con pérdida recurrente del embarazo PRE.
\end{abstract}

La importancia de los hallazgos mencionados y la novedad del presente trabajo consiste en la identificación del incremento en la respuesta autoinmune a partir del segundo aborto.

PALABRAS CLAVES: Autoanticuerpos, abortadora primaria, fosfolípido. PRE pérdida recurrente del embarazo.

SUMMARY: 90 women with two or more recurrent spontaneous abortions of unknown etiology with evidence of lack of alloimmune response and a control group of 31 women with proven fertility were evaluated for antibodies to antinuclear antigens, lupus anticoagulant and phospholipid epitopes. We confirm that autoinmune serological abnormalities are associated with recurrent pregnancy loss RPL and are detectable in patients with two spontaneous abortions.

Testing women with RPL two or more spontaneous abortions for autoantibodies to complete a panel of nuclear, lupus anticoagulant and phospholipids epitopes is recomended.

KEY WORDS: Autoantibody, primary aborter, phospholipid, RPL recurrent pregnancy loss.

\section{Introducción}

El desarrollo de la Inmunobiología de la Reproducción ha permitido un mejor entendimiento a la tolerancia del trasplante gestacional en condiciones normales. De igual manera, los avances tecnológicos logrados en los últimos años, han facilitado el conocimiento de las causas de numerosos problemas ligados íntimamente al rechazo del alo injerto feto placentario como por ejemplo la pérdida repetida del embarazo, el retardo del crecimiento intrauterino, la hipertensión inducida por el embarazo, muerte fetal, abruptio placentae, endovasculitis hemorrágica placentaria y accidentes tromboembólicos.

Para que la gestación progrese normalmente es indispensable la histoincompatibilidad entre la madre y el producto, que ha heredado del padre la mitad de su carga genética. El reconocimiento materno de la presencia del feto y el trofoblasto, (Aloinmune), por medio de diferentes antígenos de variada naturaleza, pero principalmente los del sistema HLA y especialmente el HLA-G de origen fetal, inducen una respuesta humoral, con producción de anti-

\footnotetext{
* MD Coordinador Unidad Inmunobiología y Genética.

** Instructor Asistente Colegio Mayor del Rosario.

*** Bacterióloga Coordinadora Laboratorio Unidad Inmunobiología

y Genética.

**** Bacterióloga UIBG
}

cuerpos bloqueadores, los cuales al recubrir al trasplante impiden la acción citotóxica de rechazo de la respuesta celular materna. Participan también en el estímulo del crecimiento fetal y placentario.

No están suficientemente aclarados los mecanismos que originan el daño embrionario y trofoblástico en una paciente con historia de pérdida repetida del embarazo y autoinmunidad. Los anticuerpos antifosfolípidos podrían interactuar con el endotelio vascular en los vasos deciduales causando lesión endotelial. El anticuerpo impide la liberación de ácido araquidónico por reacción cruzada con el fosfolípido en la membrana de la célula endotelial disminuyendo la producción de prostaciclina y originando fenómenos de agregación plaquetaria.

Otro mecanismo sugerido es la disminución del factor activador del plasminógeno reduciendo la conversión del plasminógeno a plasmina, alterando la lisis de fibrina. La fosfatidiletanolamina y la fosfatidilserina son moléculas de adhesión que intervienen en la capacidad de fusión celular para formar sincitio en algunos tejidos humanos, principalmente en músculo liso y en las pacientes con problemas autoinmunitarios se puede comprometer el desarrollo normal del citotrofoblasto a sincitiotrofoblasto. Adicionalmente, las vesículas normales de los fosfolípidos son estimulantes para el reconocimiento aloinmunitario necesario para el inmunotropismo y las vesículas de fosfolípidos 
agrupadas en reversa funcionan como un inmunoabsorbente natural para los anticuerpos antifosfolípidos que sabemos activan in vivo los fenómenos de hipercoagulabilidad. Por último, los lípidos de bajo peso molecular interactuan con los linfocitos $\mathrm{T}$ ayudadores bloqueando la expresión del receptor de interleukina 2 , bloqueando el proceso de activación celular dependiente de la protein-kinasa $\mathrm{C}$ y por ende bloqueando la inmunidad celular local.

De esta manera, la suma de la pobre estimulación para la protección aloinmune, la promoción de la actividad citotóxica local, el retardo en la formación del sincitio, el aumento de la agregación plaquetaria secundaria a una disminución de prostaciclina y el incremento de la producción de tromboxano por el endotelio vascular predisponen a la trombosis de los vasos deciduales. Este proceso está asociado con insuficiencia placentaria y vasculopatía decidual.

La presencia de fenómenos serológicos autoinmunes como anticuerpos antinucleares positivos, anticuerpos antifosfolípidos, anticoagulante lúpico, hipocomplementemia y o hipergamaglobulinemia están relacionadas con pérdida recurrente del embarazo PRE. (García De La Torre., 1989; Cowchock et al., 1990). Sin embargo, no se han descrito enfermedades autoinmunes específicas por estos autoanticuerpos ni se han definido con precisión los mecanismos de daño placentario y decidual. Es de resaltar no obstante que la identificación y el tratamiento para los problemas relacionados con la presencia de AAF han mejorado de manera importante el futuro obstétrico en estas pacientes. (Branch et al. 1985). Se menciona un porcentaje de nacidos vivos después del tratamiento que varía de 50 a $88 \%$, en contraste con los resultados de tratamiento con placebo cuyo rango varía de 25 a $37 \%$.

En este estudio informamos la incidencia de los anticuerpos contra seis epítopes de fosfolípidos, de anticoagulante lúpico y de anticuerpos antinucleares en pacientes con PRE de naturaleza desconocida y con patología aloinmune.

\section{Material y métodos}

\section{Selección de pacientes:}

Del primero de marzo de 1991 al treinta de octubre de 1993, 90 mujeres con antecedentes obstétricos de dos o más abortos espontáneos de naturaleza desconocida a las cuales se les diagnosticó patología aloinmune, fueron remitidas a la consulta de la Unidad de Inmunobiología y Genética para diagnóstico y tratamiento.

A todas las pacientes se les realizó una historia clínica con énfasis en la historia familiar o personal para destacar problemas alo y autoinmunes.

Ninguna paciente tenía antecedentes de trombosis o de transfusión sanguínea ni diagnóstico de lupus eritematoso sistémico o de otros desórdenes autoinmunes o coagulopatía.

La valoración clínica cuidadosa descartó problemas genéticos, anatómicos, endocrinos o infecciosos que explicaran sus antecedentes obstétricos.

Todas las parejas fueron evaluadas para etiología aloinmune con determinación del nivel de anticuerpos bloqueadores por inmunofluorescencia y en casos seleccionados por determinación de HLA. Todas las parejas presentaron un nivel de anticuerpos bloqueadores menores de $20 \%$ para células $\mathrm{T}$ y $\mathrm{B}$, se calificaron con problema de aloinmunidad para su PRE.

La calificación de abortadora primaria se realizó cuando no existía el antecedente de un parto previo.

Todos los sueros de las pacientes fueron estudiados para problemas de autoinmunidad con la detección de anticoagulante lúpico, anticuerpos antinucleares y anticuerpos de tipo Ig $\mathrm{G}$ para seis epítopes de fosfolípidos.

El grupo de control está formado por 31 mujeres sanas fértiles, sin antecedentes de abortos y sin historia personal o familiar de enfermedades autoinmunes, ni antecedentes de trombosis o de transfusión sanguínea.

\section{Estudio serológico:}

Se examinaron todos los sueros de las pacientes del estudio y grupo control para anticuerpos antinucleares (ANA), anticoagulante lúpico (ACL), anticuerpos a seis epítopes de fosfolípidos (AAF) como se describen a continuación.

\section{Anticuerpos antinucleares:}

Los anticuerpos antinucleares se midieron por técnica de inmunofluorescencia indirecta utilizando las células Hep-2 como substrato. Todos los sueros fueron examinados para actividad de ANA a la dilución 1:40. Se consideraron positivos aquellos que mostraban fluorescencia a la dilución 1:40 o superior. Las muestras positivas fueron reexaminadas a diluciones superiores progresivamente hasta encontrar el título final.

\section{Anticoagulante lúpico:}

Se estudió con la determinación de PTT, el índice de tromboplastina diluida y la prueba de neutralización de plaquetas. La presencia de 2 datos anormales se califica como positivo para la presencia de anticoagulante circulante.

\section{Anticuerpos antifosfolípidos:}

Cardiolipina, fosfatidil serina, fosfatidil inositol, fosfatidil etanolamina, fosfatidil glicerol y ácido fosfatídico de laboratorios SIGMA se prepararon para microelisa de fase sólida de acuerdo con las recomendaciones de Harris y cols en 1987. Los anticuerpos para cada epítope se estudiaron en el conjugado anti Ig G marcado con fosfatasa alcalina. Se consideraron como valores positivos las diluciones superiores a $1 / 50$.

\section{Análisis estadístico:}

Fue realizado con el programa Statistix PC DOS versión 2.0. Para comparar la edad entre los grupos de estudio y el control se utilizó la t de Student. La incidencia de autoanticuerpos se estudió con las pruebas de Chi cuadrado con la corrección de Yates y el test de Fisher exacto de acuerdo con las características de los grupos. 


\section{Resultados}

\section{Edad}

La distribución por edad para mujeres abortadoras primarias, de acuerdo al antecedente de dos, tres y cuatro o más abortos y el grupo control se presenta en la tabla 1. No se encuentran diferencias significativas entre los diferentes grupos de estudio y el control.

Tabla 1

\section{EDAD DE ABORTADORAS PRIMARIAS Y CONTROLES}

\begin{tabular}{|c|c|c|c|c|}
\hline & \multicolumn{4}{|c|}{ 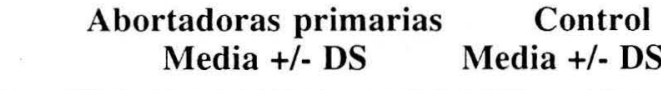 } \\
\hline & $\begin{array}{c}\mathrm{A} 2 \\
\mathrm{n}=49\end{array}$ & $\begin{array}{c}\mathrm{A} 3 \\
\mathrm{n}=29\end{array}$ & $\begin{array}{c}\mathrm{A} 4 \mathrm{y}+ \\
\mathrm{n}=12\end{array}$ & $\mathrm{n}=31$ \\
\hline Edad & $30.6+/-4.4$ & $30.7+/-4.7$ & $30.4+/-3.9$ & $26.2+1-5$ \\
\hline $\mathrm{t}$ & NS & NS & NS & \\
\hline
\end{tabular}

NS, diferencias no significativas por la t de Student.

\section{Anticuerpos antinucleares}

La incidencia de los anticuerpos antinucleares en los grupos de abortadoras primarias y controles se presenta en la tabla 2. El patrón moteado es el que se encontró con mayor frecuencia. En dos pacientes del grupo control con resultado positivo, las dos muestras se encontraron en diluciones de 1:40, en el grupo de abortadoras primarias, catorce pacientes con resultados positivos, ocho de ellos en 1:40 y los restantes positivos el mayor en 1:1280. Las pacientes con antecedente de tres abortos muestran diferencia significativa con relación al grupo control. En las pacientes con antecedente de dos o de cuatro y más abortos las diferencias no son significativas estadísticamente.

\section{Tabla 2}

LA INCIDENCIA DE ANA ENTRE ABORTADORAS PRIMARIAS Y CONTROL

\begin{tabular}{|c|c|c|c|c|}
\hline & \multicolumn{3}{|c|}{$\begin{array}{c}\text { Abortadoras primarias } \\
(\%)\end{array}$} & $\begin{array}{c}\text { Control } \\
(\%)\end{array}$ \\
\hline & $\begin{array}{c}\mathrm{A} 2 \\
\mathrm{n}=49\end{array}$ & $\begin{array}{c}\mathrm{A} 3 \\
\mathrm{n}=29\end{array}$ & $\begin{array}{c}\mathrm{A} 4 \mathrm{y}+ \\
\mathrm{n}=12\end{array}$ & $\mathrm{n}=31$ \\
\hline ANA & $\begin{array}{c}6 \\
(12.24 \%)\end{array}$ & $\begin{array}{c}7 \\
(24.13 \%)\end{array}$ & $\begin{array}{c}1 \\
(8.33 \%)\end{array}$ & $\begin{array}{c}2 \\
(6.45 \%)\end{array}$ \\
\hline $\mathrm{P}$ & $<0.40 \mathrm{NS}$ & $<0.05$ & $<0.82 \mathrm{NS}$ & \\
\hline
\end{tabular}

Se aplicó el test de Fisher exacto.

NS, no significativo.

\section{Anticoagulante lúpico}

Se presenta la incidencia de anticoagulante lúpico en los grupos de abortadoras primarias y grupo control en la tabla 3 . Las diferencias se aprecian en los tres grupos y son estadísticamente significativas para los pacientes que presentan antecedente de dos y de cuatro y más abortos al compararlas con el grupo de control. Para las noventa pruebas en el grupo de estudio se encontró positividad en $11 \%$ y en las treinta y un pruebas del control sólo dos pacientes con una de las tres pruebas alteradas, calificadas como negativas.

Tabla 3

INCIDENCIA DE ANTICOAGULANTE LUPICO EN ABORTADORAS PRIMARIAS Y CONTROL

\begin{tabular}{|c|c|c|c|c|}
\hline & \multicolumn{3}{|c|}{$\begin{array}{c}\text { Abortadoras primarias } \\
(\%)\end{array}$} & $\begin{array}{c}\text { Control } \\
(\%)\end{array}$ \\
\hline & $\begin{array}{c}\mathrm{A} 2 \\
\mathrm{n}=49\end{array}$ & $\begin{array}{c}\mathrm{A} 3 \\
\mathrm{n}=29\end{array}$ & $\begin{array}{c}\mathrm{A} 4 \mathrm{y}+ \\
\mathrm{n}=12\end{array}$ & $\mathrm{n}=31$ \\
\hline $\mathrm{ACL}$ & $\begin{array}{c}6 \\
(12.24 \%)\end{array}$ & $\begin{array}{c}2 \\
(6.89 \%)\end{array}$ & $\begin{array}{c}2 \\
(16.66 \%)\end{array}$ & 0 \\
\hline $\mathrm{P}$ & $<0.40$ & $<0.13$ & $<0.02$ & \\
\hline
\end{tabular}

Estudiado con test de Fisher exacto.

\section{Anticuerpos antifosfolípidos}

Se determinó la incidencia de anticuerpos antifosfolípidos para cada uno de los tres diferentes grupos de abortadoras primarias y en el control. Como es materia de discusión la relación que tienen los diferentes anticuerpos entre sí se investigó inicialmente la incidencia de anticuerpos a uno o más fosfolípidos de tal manera que cuando una paciente tenía por lo menos uno o más anticuerpos se consideraba positiva de manera genérica para antifosfolípidos. En la tabla 4 se presentan los resultados para abortadoras primarias según el antecedente de dos, tres y cuatro o más abortos y el grupo control. Las diferencias de los grupos con el control se encontraron estadísticamente significativas, al aplicar el test de Fisher exacto. Destacamos el valor de anticuerpos para fosfolípidos en el grupo de pacientes con antecedente de tres abortos, una cuarta parte de los pacientes presenta positividad para al menos uno de los fosfolípidos.

Se realizó luego la investigación de anticuerpos específicos para antifosfolípidos en las pacientes y los controles. Las incidencias de anticuerpos para cada grupo de abortadoras primarias y su grupo control se presentan en las tablas 5,6 y 7 con su correspondiente análisis estadístico.

$$
\text { Tabla } 4
$$

INCIDENCIA DE ANTICUERPOS ANTIFOSFOLIPIDOS: ABORTADORAS PRIMARIAS Y CONTROL

\begin{tabular}{|c|c|c|c|c|}
\hline & \multicolumn{3}{|c|}{$\begin{array}{c}\text { Abortadoras primarias } \\
(\%)\end{array}$} & $\begin{array}{c}\text { Control } \\
(\%)\end{array}$ \\
\hline & $\begin{array}{c}\mathrm{A} 2 \\
\mathrm{n}=294\end{array}$ & $\begin{array}{c}\mathrm{A} 3 \\
\mathrm{n}=174\end{array}$ & $\begin{array}{c}\mathrm{A} 4 \mathrm{y}+ \\
\mathrm{n}=72\end{array}$ & $\mathrm{n}=186$ \\
\hline $\mathrm{AAF}$ & 42 & 43 & 12 & 2 \\
& $(14.28 \%)$ & $(24.71 \%)$ & $(16.66 \%)$ & $(1.07 \%)$ \\
\hline $\mathrm{P}$ & $<0.001$ & $<0.001$ & $<0.001$ & \\
\hline
\end{tabular}

Estudiado con test de Fisher exacto.

Para el grupo con antecedente de dos abortos la tabla 5 muestra la incidencia de anticuerpos destacando que los 
dirigidos a cardiolipina, fosfatidiletanolamina y fosfatidilserina fueron significativamente mayores que el grupo control $(\mathrm{P}<0.05)$.

En la tabla 6 se aprecian los resultados para el grupo de pacientes con antecedente de tres abortos y el grupo control. Los resultados son significantes de manera estadística para todos los fosfolípidos. Los que se encuentran con mayor frecuencia positivos son cardiolipina, fosfatidilinositol y fosfatidiletanolamina.

Tabla 5

INCIDENCIA DE AAF EN PACIENTE CON ANTECEDENTE DE 2 ABORTOS Y CONTROL

\begin{tabular}{|c|c|c|c|}
\hline Antígeno fosfolipídico & $\begin{array}{c}\text { A2 } \\
(\mathbf{\%})\end{array}$ & $\begin{array}{c}\text { Control } \\
(\mathbf{\%})\end{array}$ & $\mathbf{P}$ \\
\hline Cardiolipina & 24.48 & 3.22 & $<0.02 \mathrm{Y}$ \\
\hline Etanolamina & 18.36 & 3.22 & $<0.04 \mathrm{~F}$ \\
\hline Serina & 14.28 & 0 & $<0.02 \mathrm{~F}$ \\
\hline Glicerol & 10.20 & 0 & $<0.06 \mathrm{~F}$ \\
\hline Acido fosfatídico & 10.20 & 0 & $<0.06 \mathrm{~F}$ \\
\hline Inositol & 8.16 & 0 & $<0.1 \mathrm{~F}$ \\
\hline
\end{tabular}

Y Chi cuadrado con corrección de Yates. F Fisher exacto.

Tabla 6

INCIDENCIA DE AAF EN ANTECEDENTE DE 3 ABORTOS Y CONTROL

\begin{tabular}{|c|c|c|c|}
\hline Antígeno fosfolipídico & $\begin{array}{c}\mathbf{A 3} \\
(\mathbf{\%})\end{array}$ & $\begin{array}{c}\text { Control } \\
(\mathbf{\%})\end{array}$ & $\mathbf{P}$ \\
\hline Cardiolipina & 34.48 & 3.22 & $<0.005 \mathrm{Y}$ \\
\hline Etanolamina & 24.13 & 3.22 & $<0.04 \mathrm{Y}$ \\
\hline Serina & 17.24 & 0 & $<0.01 \mathrm{~F}$ \\
\hline Glicerol & 20.69 & 0 & $<0.007 \mathrm{~F}$ \\
\hline Acido fosfatídico & 20.69 & 0 & $<0.007 \mathrm{~F}$ \\
\hline Inositol & 31.03 & 0 & $<0.0008 \mathrm{~F}$ \\
\hline
\end{tabular}

Y Chi cuadrado con corrección de Yates. F Test de Fisher exacto.

En la tabla 7 presentamos la incidencia de autoanticuerpos para el grupo de abortadoras primarias con antecedente de cuatro y más abortos. En estos resultados se aprecia que son estadísticamente significativas las diferencias con el grupo control para la cardiolipina, la fosfatidilserina y el ácido fosfatídico.

Tabla 7

INCIDENCIA DE AAF EN ANTECEDENTE DE CUATRO Y MAS ABORTOS Y CONTROL

\begin{tabular}{|c|c|c|c|}
\hline Antígeno fosfolipídico & $\begin{array}{c}\mathbf{A 4} \mathbf{y}+ \\
(\mathbf{\%})\end{array}$ & $\begin{array}{c}\text { Control } \\
(\mathbf{\%})\end{array}$ & $\mathbf{P}$ \\
\hline Cardiolipina & 33.33 & 3.22 & $<0.02 \mathrm{Y}$ \\
\hline Etanolamina & 16.66 & 3.22 & $<0.12 \mathrm{~F}$ \\
\hline Serina & 16.66 & 0 & $<0.01 \mathrm{~F}$ \\
\hline Glicerol & 8.33 & 0 & $<0.1 \mathrm{~F}$ \\
\hline Acido fosfatídico & 16.66 & 0 & $<0.01 \mathrm{~F}$ \\
\hline Inositol & 8.33 & 0 & $<0.1 \mathrm{~F}$ \\
\hline
\end{tabular}

Y Chi cuadrado con corrección de Yates. F Test de Fisher exacto.

\section{Autoinmunidad}

En los diferentes grupos de abortadoras primarias se investigaron los totales de positividad serológica a las pruebas de autoinmunidad, considerando como resultados positivos cuando se presentaba en un paciente por lo menos una de las tres pruebas practicadas y se compararon estos hallazgos con el grupo control.

Los resultados para todos los grupos son estadísticamente significativos y se presentan en la tabla 8 .

\section{Tabla 8 \\ INCIDENCIA DE AUTOANTICUERPOS EN ABORTADORAS PRIMARIAS Y CONTROL}

\begin{tabular}{|c|c|c|c|c|}
\hline & \multicolumn{3}{|c|}{$\begin{array}{c}\text { Abortadoras primarias } \\
(\%)\end{array}$} & $\begin{array}{c}\text { Control } \\
(\%)\end{array}$ \\
\hline & $\mathrm{n}=49$ & $\mathrm{n}=29$ & $\mathrm{n}=12$ & $\mathrm{n}=31$ \\
\hline $\begin{array}{c}\text { Autoanti- } \\
\text { cuerpos }\end{array}$ & $\begin{array}{c}24 \\
(49 \%)\end{array}$ & 20 & 6 & 4 \\
$(69 \%)$ & $(50 \%)$ & $(13 \%)$ \\
\hline $\mathrm{P}$ & $<0.002 \mathrm{Y}$ & $<0.0001 \mathrm{Y}$ & $<0.02 \mathrm{Y}$ & \\
\hline
\end{tabular}

Y Chi cuadrado con la corrección de Yates.

\section{Discusión}

En este estudio se presenta la incidencia de autoanticuerpos en diferentes grupos de mujeres con pérdida repetida de embarazo (con antecedentes de dos, tres y cuatro o más abortos espontáneos), con evidencia de una pobre respuesta aloinmune y quienes no presentaban enfermedades autoinmunes clínicas. El estudio corrobora previos reportes sobre la asociación entre anormalidades serológicas autoinmunes y PRE (Cowchock et al., 1986; Barbui et al., 1988; Kwack et al., 1992).

Se ha reportado que durante el embarazo normal hay activación de mecanismos de autoinmunidad y que el mismo puede servir para regular la producción de autoanticuerpos (down regulation). Este mecanismo aparentemente no opera adecuadamente en pacientes con embarazos tempranos que terminan en aborto espontáneo.

Muchos estudios han destacado el incremento del riesgo de pérdida de embarazo en relación directa con el número de pérdidas previas de embarazo (Strobino et al., 1986; Parazzini et al., 1988; Coulam, 1991). Esta tendencia está relacionada con el incremento de autoanticuerpos con cada pérdida de embarazo.

Encontramos en nuestro estudio que las pacientes con antecedente de PRE, a partir del segundo aborto presentan una mayor incidencia de anticuerpos antifosfolípidos, de ACL y de ANA con relación al grupo control, hecho absolutamente novedoso en la literatura sobre autoinmunidad y PRE porque se presentan siempre los estudios en pacientes con antecedentes de tres o más pérdidas de embarazo. La tendencia a la aparición de anticuerpos es progresiva a medida que aumentan las pérdidas del embarazo, no confirmada en el presente estudio posiblemente por el número reducido de pacientes con antecedente de cuatro o más abortos. En general se observa como la mitad de las pacientes con PRE presentan alguna evidencia de autoanticuerpos en comparación con un $13 \%$ del grupo control. 
Como un hecho clínico destacable es la investigación en las pacientes con antecedente de dos abortos, porque de manera tradicional se ha postulado que la posibilidad de problemas inmunológicos asociados con la PRE se hace evidente después de la tercera pérdida, donde se sugiere puede encontrarse como factor etiológico hasta en $40 \%$ de los pacientes. Nuestro estudio nos reafirma la necesidad de investigar de una forma más oportuna, a partir de la segunda pérdida a estas pacientes.
Se encuentra la mayor incidencia para los anticuerpos dirigidos a cardiolipina, fosfatidiletanolamina, fosfatidilinositol y fosfatidilserina, razón por la cual el estudio del panel de seis fosfolípidos es totalmente necesario.

Destacamos la relevancia del estudio de autoinmunidad en estas pacientes dentro de su valoración clínica y consideramos, en estudios posteriores, la cuantificación de los niveles de anticuerpos y el estudio de los isotipos Ig M e Ig A.

\section{BIBLIOGRAFIA}

1. Coulam C., Faulk P., McIntyre JA., Critser JK. Pregnancy outcome after diagnosis of recurrent spontaneous abortion. In: Mettler L, Billington WD, eds. Reproductive Inmunology 1989. Amsterdam: Elsevier Science Publishers 1990; 195-202.

2. Ober C., Elias S. The role of HLA-Dr in reproduction: results of prospective, population-based studies in U.S. Hutterites In: Mettler L, Billington WD, eds. Reproductive Inmunology 1989. Amsterdam: Elsevier Science Publishers 1990; 203-210.

3. Mowbray JF., Underwood JL. Inmunization with paternal cells in treatment of very early recurrent abortion. In: Mettler 1; Billington WD, eds. Reproductive Inmunology 1989. Amsterdam: Elsevier Science Publishers 1990; 211-216.

4. Rote NS, NG AK., Ault KA., Carmody M., Dostal-Johnson DA., Mitchell J,NG S., Roberts R., Siekman R. Antiphospholipid antibodies and recurrent pregnancy loss: Analysis of monoclonal and polyclonal antiphosphatidylserine antibodies. In: Mettler L, Billington WD, eds. Reproductive Inmunology 1989. Amsterdam: Elsevier Science Publishers 1990; 217-226.

5. Beer AE. Inmunologic aspects of normal pregnancy and recurrent spontaneous abortion. Semin. Reprod. Endo. 1988; 6: 163-180.

6. Beer AE., Quebbeman JF., Ayers JWT., Haines RF. Major histocompatibility complex antigens, maternal and paternal inmune responses, and chronic habitual abortions in human. Am. J. Obstet. Gynecol. 1981; 141: 987-999.

7. Sibai BM., Mirro R., Chesney CM., Leffler C. Low dose aspirin in pregnancy. Obstet. Gynecol. 1898; 74: 551-557.

8. McIntyre JA., Faulk WP., Nichols-Johnson VR., Taylor CG. Inmunologic testing and inmunotherapy in recurrent spontaneous abortion. Obstet. Gynecol. 1986; 67: 169.

9. Beer AE. New horizons in the diagnosis, evaluation and therapy of recurrent spontaneous abortion. Clin. Obstet. Gynecol. 1986; 13: 115-122.

10. Ho H-N., Gill TJ., Nsieh R-P., Hsieh H-J., Lee T-Y. Sharing of human leukocyte antigens in primary and secondary recurrent spontaneous abortions. Am. J. Obstet. Gynecol 1990; 163: 178-188.

11. Branch DW., Andres R., Digre KB., Rote NS., Scott JR. The association of antiphospholipid antibodies with severe preeclampsia. Obstet. Gynecol. 1989; 73: 541-545.

12. Chavez D., McIntyre JA., Colliver JA., Faulk WP. Allogeneic matings and inmunization have diferent effects on nulliparous and multiparous mice. J. Inmunol. 1987; 139: 85-88.

13. Redman CWG. Inmunology of the placenta. Clin. Obstet. Gynecol. 1986; 13: 469-499.

14. Billington RE. From transplantation biology to reproductive inmunobiology. Obstet. Gynecol. 1978; 7: 1-14.

15. Kovats S., Main EK., Librach C., Stubblebine M., Fisher S., DeMars R. A class I antigen, HLA-G, expressed in Human trophoblasts. Science 1990; 248: 220-223.

16. Infante-Rivard C., David M., Gauthier R., Rivard G. Lupus anticoagulants, anticardiolipin antibodies, and fetal loss. N. Engl. J. Med. 1991; 325: 1063-1066.

17. Kwak JYH., Beer AE. Reproductive outcome in women with recurrent spontaneous abortions of alloinmune and autoinmune etiologies; pre vs post conceptional treatment. Dept. of Ob/Gyn, UHS/Chicago Medical School, North Chicago, IL 60064.

18. Kwak JYH., Gilman-Sachs A., Beaman KD., Beer AE. Autoantibodies in women with primary recurrent spontaneous abortion (RSA) of alloinmune etiology. Dept of Ob/Gyn, UHS/Chicago Medical School, North Chicago, IL 60064.
19. Barini, Kwak J, Polgar K., Hill JA., Beer AE. The embryotoxic factor assay in women with recurrent pregnancy loss and subsequent pregnancy outcome. Dept. of Ob/Gyn, UHS/Chicago Medical School, North Chicago, IL 60064 and Harvard Medical School, Boston, MA 02115.

20. Salafia C., Barini R., Vogel C., Kwak J., Beer AE. Placental inmunopathology in live born infants of women with recurrent spontaneous abortions (RSA) of alloinmune etiology inmunized with paternal limphocytes. Dept. of Ob/Gyn, UHS/Chicago Medical School, North Chicago, IL 60064 and Danbury Hospital, Danbury CT.

21. Kwak J., Ober C., Barini R., Beer AE. Maternal autoinmune abnormalities and fetal HLA-DQA1 alleles in women with recurrent spontaneous abortion (RSA). Dept. of Ob/Gyn, UHS/Chicago Medical School, North Chicago, IL 60064.

22. Gurka G., Rocklin R. Reproductive Inmunology. JAMA 1987;258: 2983-2987.

23. Hanson LA., Carlsson B., Ekre HP., Hahn-Zoric M., Osterhaus AD., Roberton D. Inmunoregulation mother fetus/newborn, a role for antiidiotypic antibodies. Acta Pediatr Scand 1989; 351: 38-41.

24. Crainie M., Stimson WH. The maternal inmune response to a new human trophoblast antigen. J. Clin. Lab. Inmunol. 1988; 27: 103-107.

25. Claman HN. The biology of the inmune response. JAMA $1987 ; 258$ : 2834-2840.

26. Scott Jr., Rote NS., Branch DW. Inmunologic aspects of recurrent abortion and fetal death. Obstet. Gynecol 1987; 70: 645-656.

27. Clark D., Daya S. Inmunotherapy for unexplaneid recurrent abortion; problems in proving efficacy/inefficacy. Am. J. Reprod. Inmunol. 1991; 25: 49.

28. Ho H-N., Gill TJ., Hsieh C-Y, Lee T-Y. Inmunotherapy for recurrenı spontaneous abortions in a chinese population. Am. J. Reprod. Inmunol. 1991; 25: 49.

29. Johnson P., Ramsden G. A combined randomized double blind and open study of trophoblast membrane infusion (TMI) in unexplained recurrent abortion. Am. J. Reprod. Inmunol 1991; 25: 50.

30. Huetz F., Jacquemart F., Peña C., Varela F., Coutinho A. Autoinmunity: the moving boundaries between physiology and pathology. In Bach JF eds. Inmunointervention in autoinmune diseases. London: Academic Press Limited 1989; 11-22.

31. Hill J., Polgar K., Harlow B., Anderson D. Evidence of embryo and trophoblast-toxic cellular immune response(s) in women with recurrent spontaneous abortion. Am. J. Obstet. Gynecol. 1992; 166: 10441052.

32. Ladines C., Maruo T., Manalo A., Mochizuki M. Cytologic localization of epidermal growth factor and its receptor in developing human placenta varies over the course of pregnancy. Am. J. Obstet. Gynecol. 1991; 165: 1377-1382.

33. Hansen K., Opsahi M., Nieman L., Baker J., Klein T. Natural killer cell activity from pregnant subjects is modulated by RU 486. Am. J. Obstet. Gynecol 1992; 166: 87-90.

34. Silver R., Adler L., Hickman, Hageman J. Anticardiolipin antibody positive serum enhances endothelial cell platelet activating factor production. Am. J. Obstet. Gynecol. 1991; 165: 1748-1752.

35. Feinberg B., Tan N., Gonik B., Brath P., Walsh S. Increased progesterone concentrations are necesary to suppress interleukin 2 activated human mononuclear cell cytotocity. Am. J. Obstet. Gynecol. 1991; 165: 1872-1875. 